pISSN 2466-1384 eISSN 2466-1392

大韓獸醫學會誌 (2018) 第 58 卷 第 2 號

Korean J Vet Res(2018) 58(2) : 99 101

https://doi.org/10.14405/kjvr.2018.58.2.99

$<$ Case Report $>$

\title{
Imaging diagnosis of hepatic lipidosis in a cat
}

\author{
Seong-Hun Heo, Young-Min Yoon, Tae-Sung Hwang, Dong-In Jung, Hee-Chun Lee* \\ Institute of Animal Medicine, College of Veterinary Medicine, Gyeongsang National University, Jinju 52828, Korea
}

(Received: March 7, 2018; Revised: March 22, 2018; Accepted: April 5, 2018)

\begin{abstract}
A 5-year-old intact female Persian cat was referred with anorexia and vomiting. Physical examination revealed icterus in the pinna, oral mucosa, and sclera. Ultrasonography revealed several findings including uniformly hyperechoic hepatic parenchyma compared to adjacent falciform fat, increased attenuation of the ultrasound beam, and poor visualization of intrahepatic vessel borders. Computed tomography revealed hypoattenuation of the hepatic parenchyma with a radiodensity value of -60 Hounsfield units. The adjacent intrahepatic vessels appeared hyperattenuated relative to the hepatic parenchyma as if the vessels were contrast-enhanced. Based on ultrasonography and computed tomography results, the cat was tentatively diagnosed as feline hepatic lipidosis.
\end{abstract}

Keywords: cats, computed tomography, fatty liver, lipidoses, ultrasonography

Feline hepatic lipidosis (FHL) is most common liver diseases in cats caused by excessive accumulation of triglycerides in hepatocytes that induce cholestasis and hepatic dysfunction [15]. It was initially known as idiopathic condition, but now most of the affected cats are found to have a disease or environment that directly cause malnutrition [2]. The following several ultrasonographic criteria were associated with severe hepatic lipidosis: the hyperechoic liver compared with falciform fat; the isoechoic or hyperechoic liver compared with omental fat, poor visualization of intrahepatic vessel borders and increased attenuation of the ultrasound beam. The most useful criteria was the diffusely hyperechoic hepatic parenchyma compared with falciform fat and believed to be a highly sensitive and specific diagnostic indicator [14]. However, a recent study reported that the hepatic parenchyma could have diffusely hyperechoic compared with falciform fat in clinically normal obese cats [10]. Additionally, other diseases such as lymphoma, and hepatic cirrhosis/fibrosis also have been described as a diffusely hyperechoic liver [9]. Fat is radiolucent compared to other tissues and fat-rich organs are described lower attenuation with computed tomography (CT). It is a unique feature of fat that can be helpful for differentiation from other pathological changes $[6,8,13]$.

A 5-year-old intact female Persian cat was referred to Gyeongsang National University Veterinary Medical Teaching Hospital with anorexia and vomiting. The cat used to be obese but had a history of sudden weight loss from 6.6 to $4 \mathrm{~kg}$ for a month. Physical examination revealed icterus in the pinna, oral mucosa, and sclera. A weak pain is elicited on manipulation of the abdomen. Complete blood cell counts was within normal ranges. On the biochemical profile, abnormalities were noted as follows; increased alkaline phosphatase (ALP) $225 \mathrm{U} / \mathrm{L}$ (reference range, 10 to $90 \mathrm{U} / \mathrm{L}$ ), increased alanine aminotransferase (ALT) $431 \mathrm{U} / \mathrm{L}$ (reference range, 20 to $100 \mathrm{U} / \mathrm{L}$ ), increased bile acid $113 \mathrm{U} / \mathrm{L}$ (reference range, 0 to $25 \mu \mathrm{mol} / \mathrm{L}$ ), increased total bilirubin $8.9 \mathrm{mg} / \mathrm{dL}$ (reference range, 0.1 to $1.6 \mathrm{mg} / \mathrm{dL}$ ), and decreased blood urea nitrogen $5 \mathrm{mg} / \mathrm{dL}$ (reference range, 10 to $30 \mathrm{mg} / \mathrm{dL}$ ).

Radiography did not reveal remarkable findings. Ultrasonography reveals uniformly increased hepatic parenchymal echogenicity including hyperechoic hepatic parenchyma compared to adjacent falciform fat, increased attenuation of the ultrasound beam, poor visualization of intrahepatic vessel borders, a blunt margin of the hepatic lobe and the hourglass-shaped gallbladder (GB) (Fig. 1). CT revealed a severely hypoattenuated hepatic parenchyma with a radiodensity value of -60 Hounsfield units (HU) and normal spleen with a radiodensity value of $51 \mathrm{HU}$. Adjacent intrahepatic vessels appear hyperattenuated relative to the hepatic parenchyma as if the vessels were contrast-enhanced (Fig. 2). The hourglassshaped GB that had already been revealed by ultrasonography was also found.

Based on ultrasonography and CT findings, the cat was tentatively diagnosed as the FHL, and the biopsy was not performed. Regular follow-up was performed weekly with the treatment. As the treatment progressed, on ultrasonography, the echogenicity and echotexture of the hepatic paren-

*Corresponding author

Tel: +82-55-772-2356, Fax: +82-55-772-2330

E-mail: 1hc@gnu.ac.kr 

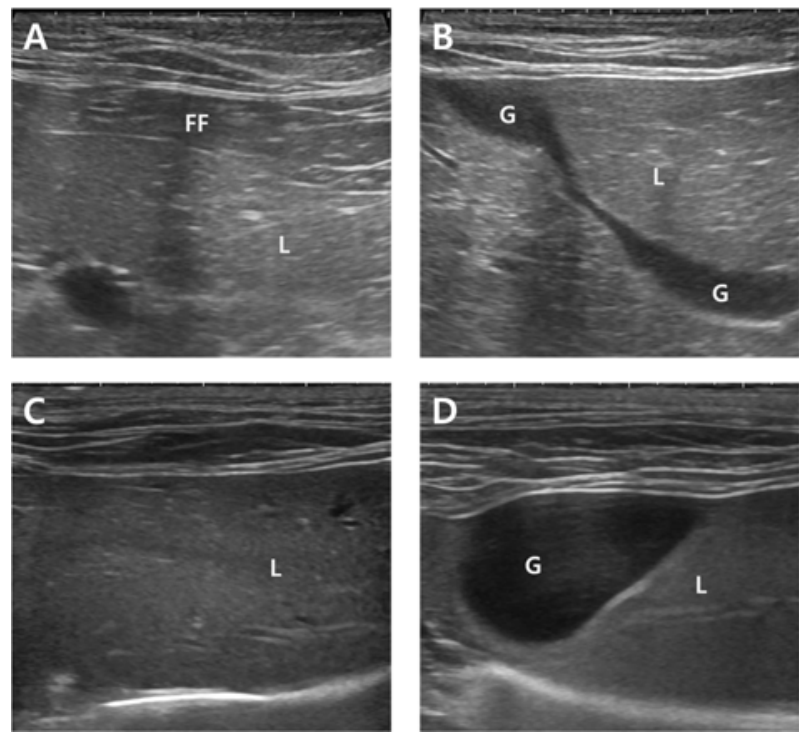

Fig. 1. Ultrasonographic images of the liver and gallbladder. (A) Hyperechoic hepatic parenchyma compared with falciform fat, increased diffuse the ultrasound beam, poor visualization of intrahepatic vessel borders are present. (B) The gallbladder appears hourglass-shape because of circumscribed protrusion of hepatic parenchyma. (C and D) Hepatic parenchymal echogenicity and gallbladder were recovered to normal after treatment. FF, falciform; L, liver; G, gallbladder.

chyma gradually became normal and hourglass-shaped GB gradually became round shape. No significant changes were found in radiography. The cat's body weight gradually was increased, and all of the clinical symptoms, blood tests also recovered to normal.

FHL occurs primarily in middle-aged to older cats and there is no known gender or breed predilections [1]. Most cats affected with FHL are obese housecats which have undergone a period of anorexia. Physical examination findings usually reveal icterus, hepatomegaly, dehydration, and a loss of at least $25 \%$ of body weight [1]. In this case, the cat had a typical appearance of FHL. The cat was a 4-year-old obese housecat and had a history of anorexia for a month. Physical examination also revealed marked icterus and weight loss ( $40 \%$ reduction).

Clinicopathologic findings in FHL are characterized by intrahepatic cholestatic disorder and involved increased serum bilirubin, ALP and ALT. Especially, primary FHL case tends to have higher ALP activity and higher total bilirubin than secondary FHL case, and tend to have low gammaglutamyl transpeptidase activity and absence of hyperglobulinemia because it is not associated with other hepatobiliary diseases [3]. In this case, the cat was more likely to have primary FHL.

Radiography in a patient with FHL often reveals hepatomegaly related to infiltration of triglycerides in hepatocytes but it is an inconsistent and non-specific finding for diagnosis [1]. In humans, fatty liver can be accurately assessed
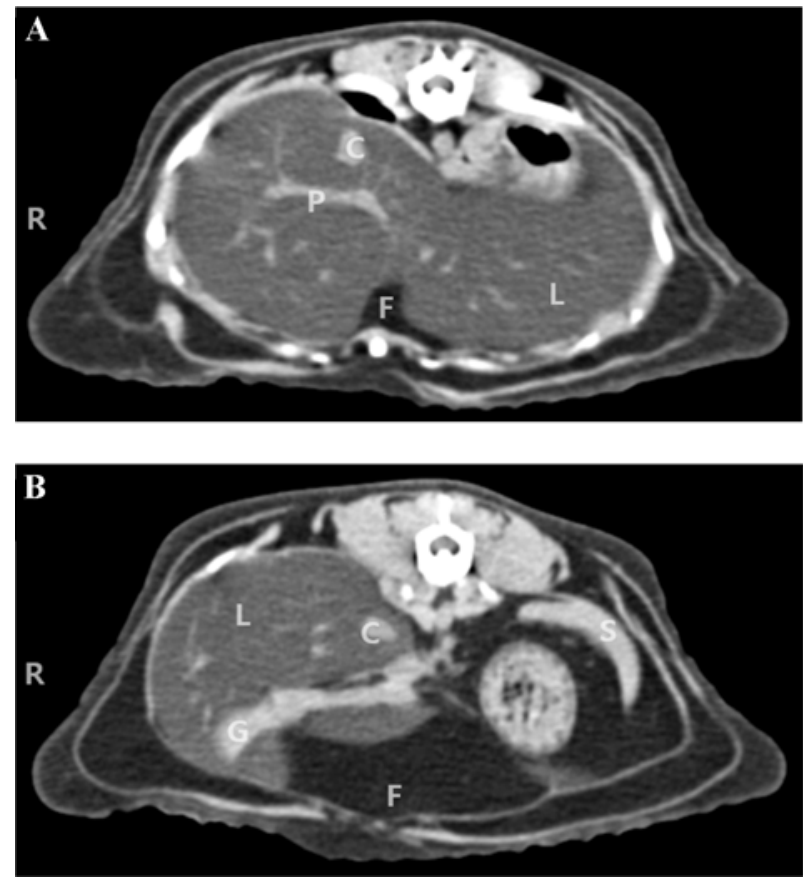

Fig. 2. Transverse abdominal pre-contrast computed tomography images of the cat. (A) Diffuse hypoattenuation of the hepatic parenchyma ( -60 Hounsfield units) is evident and adjacent intrahepatic vessels are apparently hyperattenuated as if the vessels contrast-enhanced. (B) The compressed gallbladder because of circumscribed protrusion of the hepatic parenchyma. $\mathrm{C}$, caudal vena cava; P, portal v; F, falciform fat; L, liver; S, spleen.

through radiography only if the patients have moderate or severe fat deposition [11]. In this case, there was no radiographic finding that indicating FHL or other diseases. No radiographic significant changes were also found after the cat recovered.

Based on ultrasonography, diffusely increased hepatic echogenicity has been reported with FHL, obesity, fibrosis, and lymphoma in the cat $[9,10,14]$. One study suggested that the liver could have hyperechoic than falciform ligament in normal obese cats makes complicates the ultrasonographic diagnosis of FHL because the obese cats are predisposed to develop FHL [10]. In human, patterns of increased echogenicity of hepatic parenchyma have been termed the "fattyfibrotic pattern" to emphasize the inability of ultrasonography to distinguish distinctly fatty change from fibrosis and the possibility that two lesions may be often concurrent [12]. Similarly, one study reported that hepatic fibrosis was diagnosed histologically in 6-11\% of cats with FHL [4]. However, a recent study reported that increased hepatic echogenicity was related to the degree of fat infiltration but not to the degree of fibrosis or cirrhosis, and so is posterior beam attenuation [7]. Moreover, the fine echo pattern of the FHL and the coarse echo pattern of the fibrosis were reported critical criteria for distinguishing them [5]. In this case, echotexture of hepatic parenchyma was extremely fine and the possibility of fibro- 
sis was low. These findings were consistent with FHL, but other diseases could not be excluded completely.

In the veterinary medicine, CT quantification of the liver in healthy cats has been reported in two studies. One study reported that the mean hepatic attenuation was $54.7 \pm 5.6 \mathrm{HU}$ (mean \pm SD, ranged from 42.7 to 64.8 ) in 26 normal cats [8]. Another study reported that the mean hepatic attenuation was $70.7 \pm 8.7 \mathrm{HU}$ (mean $\pm \mathrm{SD}$, ranged from 53.4 to 88.1) in 30 normal cats, but the author discussed that the result could be 11-24 HU higher than the previous report due to the difference in performance of the CT scanner [6]. In the present case, the attenuation of the hepatic parenchyma was $-60 \mathrm{HU}$, which was about $110 \mathrm{HU}$ difference compared to the normal variation and almost the same as the value of fat ranging from -80 to $-100 \mathrm{HU}$. The intrahepatic vessels that appear hyperattenuated relative to the hepatic parenchyma suspected severe fat deposition. Lipomas and infiltrative lipoma were also known to have severe homogeneous hypoattenuation (about $-110 \mathrm{HU}$ ) in CT, but both have been found as focal or multifocal lesions [13]. Based on these CT findings, FHL was tentatively diagnosed even though the cut-off value of radiodensity for diagnosing FHL has not been established yet.

Conclusively, in this case, FHL was tentatively diagnosed by the noninvasive imaging method including ultrasound and CT. Above all, the hypoattenuation of the hepatic parenchyma on non-contrast CT was a significant finding for diagnosis of FHL. It is expected that if the cut-off value and the formula are established in the follow-up study, the usefulness of the CT will be increased not only in the severe FHL but also in the mild FHL.

\section{References}

1. Armstrong PJ, Blanchard G. Hepatic lipidosis in cats. Vet Clin North Am Small Anim Pract 2009, 39, 599-616.

2. Center SA. Feline hepatic lipidosis. Vet Clin North Am Small Anim Pract 2005, 35, 225-269.

3. Center SA, Crawford MA, Guida L, Erb HN, King J. A retrospective study of 77 cats with severe hepatic lipidosis:
1975-1990. J Vet Intern Med 1993, 7, 349-359.

4. Gagne JM, Weiss DJ, Armstrong PJ. Histopathologic evaluation of feline inflammatory liver disease. Vet Pathol 1996, 33, 521-526.

5. Kooby DA, Fong Y, Suriawinata A, Gonen M, Allen PJ, Klimstra DS, DeMatteo RP, D'Angelica M, Blumgart LH, Jarnagin WR. Impact of steatosis on perioperative outcome following hepatic resection. J Gastrointest Surg 2003, 7, 10341044.

6. Lam R, Niessen SJ, Lamb CR. X-ray attenuation of the liver and kidney in cats considered at varying risk of hepatic lipidosis. Vet Radiol Ultrasound 2014, 55, 141-146.

7. Mathiesen UL, Franzén LE, Åselius H, Resjö M, Jacobsson L, Foberg U, Frydén A, Bodemar G. Increased liver echogenicity at ultrasound examination reflects degree of steatosis but not of fibrosis in asymptomatic patients with mild/moderate abnormalities of liver transaminases. Dig Liver Dis 2002, 34, 516-522.

8. Nakamura M, Chen HM, Momoi Y, Iwasaki T. Clinical application of computed tomography for the diagnosis of feline hepatic lipidosis. J Vet Med Sci 2005, 67, 1163-1165.

9. Newell SM, Selcer BA, Cornelius LM. Imaging techniques for evaluating feline hepatobiliary disease. Vet Med (1985) 1994, 89, 859-864.

10. Nicoll RG, O'Brien RT, Jackson MW. Qualitative ultrasonography of the liver in obese cats. Vet Radiol Ultrasound 1998, 39, 47-50.

11. Saadeh S, Younossi ZM, Remer EM, Gramlich T, Ong JP, Hurley M, Mullen KD, Cooper JN, Sheridan MJ. The utility of radiological imaging in nonalcoholic fatty liver disease. Gastroenterology. 2002, 123, 745-750.

12. Siegelman ES, Rosen MA. Imaging of hepatic steatosis. Semin Liver Dis. 2001, 21, 71-80.

13. Spoldi E, Schwarz T, Sabattini S, Vignoli M, Cancedda S, Rossi F. Comparisons among computed tomographic features of adipose masses in dogs and cats. Vet Radiol Ultrasound 2017, 58, 29-37.

14. Yeager AE, Mohammed H. Accuracy of ultrasonography in the detection of severe hepatic lipidosis in cats. Am J Vet Res 1992, 53, 597-599.

15. Zawie DA, Garvey MS. Feline hepatic disease. Vet Clin North Am Small Anim Pract 1984, 14, 1201-1230. 